\title{
Neutron magnetic polarizability with Landau mode operators
}

\author{
Ryan Bignell, ${ }^{*}$ Jonathan Hall, Waseem Kamleh, and Derek Leinweber \\ Special Research Centre for the Subatomic Structure of Matter (CSSM), \\ Department of Physics, University of Adelaide, Adelaide, South Australia 5005, Australia \\ Matthias Burkardt \\ Department of Physics, New Mexico State University, Las Cruces, New Mexico 88003-001, USA
}

(Received 27 April 2018; published 21 August 2018)

\begin{abstract}
The application of a uniform background magnetic field makes standard quark operators utilizing gaugecovariant Gaussian smearing inefficient at isolating the ground state nucleon at nontrivial field strengths. In the absence of QCD interactions, Landau modes govern the quark energy levels. There is evidence that residual Landau mode effects remain when the strong interaction is turned on. Here, we introduce novel quark operators constructed from the two-dimensional $U(1)$ Laplacian eigenmodes that describe the Landau levels of a charged particle on a periodic finite lattice. These eigenmode-projected quark operators provide enhanced precision for calculating nucleon energy shifts in a magnetic field. Using asymmetric source and sink operators, we are able to encapsulate the predominant effects of both the QCD and QED interactions in the interpolating fields for the neutron. The neutron magnetic polarizability is calculated using these techniques on the $32^{3} \times 64$ dynamical QCD lattices provided by the PACS-CS Collaboration. In conjunction with a chiral effective-field theory analysis, we obtain a neutron magnetic polarizability of $\beta^{n}=2.05(25)(19) \times 10^{-4} \mathrm{fm}^{3}$, where the numbers in parentheses describe statistical and systematic uncertainties.
\end{abstract}

DOI: 10.1103/PhysRevD.98.034504

\section{INTRODUCTION}

The study of the magnetic polarizability of the neutron is an area of ongoing experimental and theoretical interest. Measurement of this quantity remains challenging with considerable uncertainties [1-3], although improvement has been seen in recent years [4]. There is scope for lattice QCD to make important predictions in this area.

The approach used here to calculate this quantity on the lattice is the uniform background-field method [5-7]. A $U(1)$ phase factor on the gauge links induces an external magnetic field across the entirety of the lattice. The external field causes an energy shift from which the magnetic polarizability can be determined by use of the energy-field relation [6-11]

$$
E(B)=m+\vec{\mu} \cdot \vec{B}+\frac{|q e B|}{2 m}-\frac{4 \pi}{2} \beta B^{2}+\mathcal{O}\left(B^{3}\right),
$$

where $m$ is the mass and $\vec{\mu}$ and $\beta$ are the magnetic moment and magnetic polarizability respectively. Note that the $|q e B| / 2 m$ term corresponds to the lowest Landau energy

*ryan.bignell@adelaide.edu.au

Published by the American Physical Society under the terms of the Creative Commons Attribution 4.0 International license. Further distribution of this work must maintain attribution to the author(s) and the published article's title, journal citation, and DOI. Funded by SCOAP ${ }^{3}$. and is only present for charged hadrons. There is in principle a tower of Landau levels, $(2 n+1)|q e B| / 2 m$ for $n=$ $0,1,2, \ldots[12]$.

At first glance, the method is simple; we can fit the linear and quadratic coefficients of the resulting energies as a function of field strength to extract the magnetic moment and polarizability $[6,9]$. However, baryon correlation functions suffer from a rapidly decaying signal-to-noise problem [13]. This makes the extraction of the magnetic polarizability using standard nucleon interpolating fields challenging as it appears at second order in the energy expansion, as demonstrated by previous studies [7,9-11].

The application of three-dimensional gauge-covariant Gaussian smearing on the quark fields at the source and/or sink is highly effective at isolating the nucleon ground state in pure QCD calculations. However, the presence of a uniform magnetic field alters the physics, breaking threedimensional spatial symmetry and introducing electromagnetic perturbations into the dynamics of the charged quarks.

Under a uniform magnetic field, in the absence of QCD interactions, each quark will have a Landau energy proportional to its charge. When QCD interactions are enabled, the quarks will hadronize, such that (in the confining phase) the Landau energy corresponds to that of the composite particle. In particular, as the neutron has zero charge, the $d d u$ quarks must combine such that the overall Landau energy vanishes. 
It is clear that the QCD and magnetic interactions compete with each other in the confining phase. Indeed, there is evidence that residual Landau mode effects remain when the strong interaction is turned on $[10,14]$. Here, we explore the idea of using quark operators on the lattice that capture both of these forces, choosing asymmetric source and sink operators to provide better overlap with the energy eigenstates of the neutron in a background magnetic field.

At the source, we capture the QCD dynamics by using spatial Gaussian smearing, tuned to maximise overlap with the nucleon ground state at zero magnetic-field strength. At the sink, we seek to encode the physics associated with the magnetic field by using a projection operator constructed from the eigenmodes associated with the quark lattice Landau levels. As discussed in Sec. III B, these Landau modes correspond to the eigenmodes of the two-dimensional $U(1)$ lattice Laplacian [15]. Calculations are performed at multiple quark masses in order to enable a chiral extrapolation to the physical regime.

\section{BACKGROUND FIELD METHOD}

To simulate a constant magnetic field along a single axis, the background-field method is used [5]. To derive this technique on the lattice, consider the continuum case. In the continuum, a minimal electromagnetic coupling is added to form the covariant derivative

$$
D_{\mu}=\partial_{\mu}+\text { iqe } A_{\mu}
$$

Here, $A_{\mu}$ is the electromagnetic four potential, and $q e$ is the charge on the fermion field. The equivalent modification on the lattice is to multiply the usual gauge links by an exponential phase factor,

$$
U_{\mu}(x) \rightarrow U_{\mu}(x) e^{i a q e A_{\mu}(x)}
$$

A uniform magnetic field along the $\hat{z}$ axis is obtained (in the continuum) using

$$
\begin{gathered}
\vec{B}=\nabla \times \vec{A} \\
B_{z}=\partial_{x} A_{y}-\partial_{y} A_{x},
\end{gathered}
$$

which does not uniquely specify the electromagnetic potential. The choice made over the interior of the lattice is $A_{x}=-B y$. This gives a constant magnetic field of magnitude $B$ in the $+\hat{z}$ direction. In order to maintain the constant magnetic field across the edges of the lattice where periodic boundary conditions are in effect, we set $A_{y}=$ $+B N_{y} x$ along the boundary in the $\hat{y}$ dimension. This then induces a quantization condition for the uniform-magneticfield strength [10]

$$
q e B a^{2}=\frac{2 \pi k}{N_{x} N_{y}} .
$$

Here, $a$ is the lattice spacing, $N_{x}$ and $N_{y}$ are the spatial dimensions of the lattice, and $k$ is an integer specifying the field quanta in terms of the minimum field strength.

In this work, the field quanta $k$ is set in units of the charge of the down quark, i.e., $q=-1 / 3$. Hence, a field with $k_{d}=1$ will be in the $-\hat{z}$ direction and aligned with spin-down components.

\section{QUARK OPERATORS}

We explore the use of asymmetric source and sink operators in order to construct zero-momentum projected correlation functions which have greater overlap with the energy eigenstates of the neutron in a background magnetic field. This allows us to emulate the dominant QCD and magnetic effects separately.

We consider fixed boundary conditions in the time direction and place the source at $N_{t} / 4=16$.

\section{A. Gaussian smeared source}

A smeared source is used to provide a representation of the QCD interactions, while the sink is used to capture the physics associated with the magnetic field. Several levels of source smearing are investigated at $B=0$ in order to isolate the QCD nucleon ground state. For $m_{\pi}=411 \mathrm{MeV}, 300$ sweeps of standard Gaussian smearing is optimal, as illustrated in Fig. 1. An identical process is followed at each of the quark masses producing optimal smearings of $N_{s m}=150,175,300,350$ for masses $m_{\pi}=702,570,411$, $296 \mathrm{MeV}$ respectively.

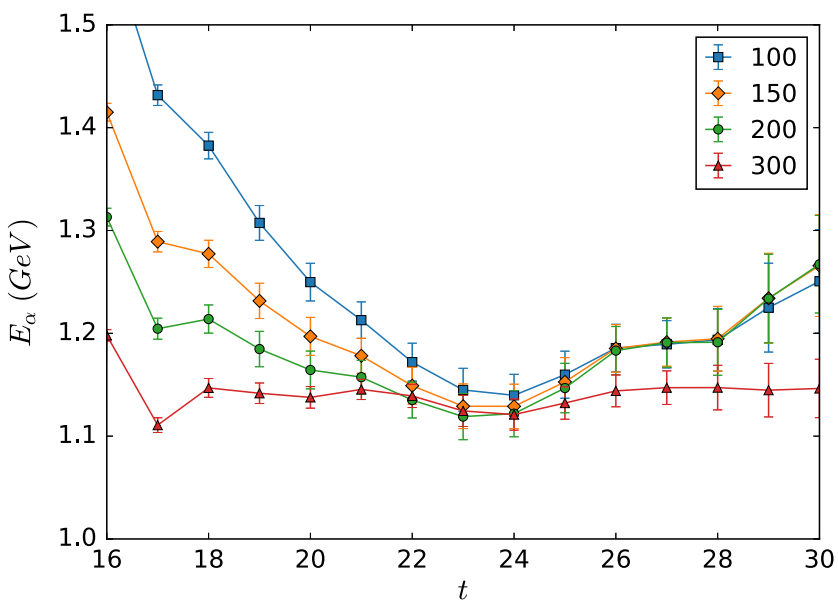

FIG. 1. Neutron zero-field effective mass from smeared source to point sink correlators for various levels of covariant Gaussian smearing at the source. The source is at $t=16$. 


\section{B. Landau mode quark sink}

In the absence of QCD interactions, the charged quarks will each have an associated Landau level. To capture the physics associated with the uniform-background magnetic field, we apply a quark level $U(1)$ Landau mode projection at the sink. To motivate this sink projection, we briefly review the relevant Landau mode physics and the relation to the continuum Dirac equation. A charged scalar particle which sits in a uniform magnetic field will have an associated Landau energy which is proportional to its charge. In the nonrelativistic approximation, the energy spectrum of a charged particle in a constant magnetic field along the $\hat{z}$ direction is equivalent to that of a harmonic oscillator, $E_{n}=\left(n+\frac{1}{2}\right) \omega$, where $\omega=|q e B| / m$ is the classical cyclotron frequency. In the infinite-volume limit, each energy level is infinitely degenerate.

The relativistic generalization of the Landau energy levels for a fermion commences with the Dirac operator coupled to electromagnetism,

$$
\not D=\gamma^{\mu} D_{\mu}=\gamma^{\mu}\left(\partial_{\mu}+i q e A_{\mu}\right) .
$$

The second-order equation for a Dirac spinor $\psi$ is

$$
\left(D^{2}+\frac{1}{2} q e \sigma^{\mu \nu} F_{\mu \nu}+m^{2}\right) \quad \psi=0,
$$

such that for a constant background magnetic field $\vec{B}$ (and in a suitable spinorial representation) we have

$$
\left(D^{2}+q e\left[\begin{array}{cc}
\vec{\sigma} \cdot \vec{B} & 0 \\
0 & \vec{\sigma} \cdot \vec{B}
\end{array}\right]+m^{2}\right) \quad \psi=0 .
$$

Choosing $\vec{B}=B \hat{z}$ in the $\hat{z}$ direction and introducing a spinpolarization factor, $\alpha= \pm 1$, the equation for each spinor component $\psi_{\tau}$ is

$$
\left(D^{2}+\alpha q e B+m^{2}\right) \psi_{\tau}=0,
$$

with $\alpha=(-1)^{(\tau-1)}$. The eigenenergies as a function of the mass, $m$; field strength, $B$; spin polarization, $\alpha$; and momentum in the $z$ direction, $p_{z}$, are given by [12]

$$
E^{2}(B)=m^{2}+|q e B|(2 n+1-\alpha)+p_{z}^{2},
$$

with $n$ describing the quantized energy level, that is, the relativistic Landau energy. The key point here is that, while the eigenenergies depend on the spin-coupling term, the basis of eigenmodes of the operator $\left(D^{2}+\alpha q e B+m^{2}\right)$ is independent of the constant terms $\alpha q e B$ and $m^{2}$, depending only on the covariant Laplacian $D^{2}=D^{\mu} D_{\mu}$. Hence, on a discrete lattice, the Landau modes for a charged Dirac particle with $\vec{B}=B \hat{z}$ correspond to the eigenmodes of the two-dimensional $U(1)$ gauge-covariant lattice Laplacian
$\Delta_{\vec{x}, \vec{x}^{\prime}}=4 \delta_{\vec{x}, \vec{x}^{\prime}}-\sum_{\mu=1,2} U_{\mu}^{B}(\vec{x}) \delta_{\vec{x}+\hat{\mu}, \vec{x}^{\prime}}+U_{\mu}^{B^{\dagger}}(\vec{x}-\hat{\mu}) \delta_{\vec{x}-\hat{\mu}, \vec{x}^{\prime}}$

where $U_{\mu}^{B}(\vec{x})$ contains the same $U(1)$ phases as applied in the full lattice QCD calculation. On a finite-volume lattice, the degeneracy of the lattice Landau modes is finite and is dependent on the product $q e B$ of the charge and magneticfield strength. This is in contrast to the infinite degeneracy of the infinite volume. In particular, the lowest Landau level on the lattice has a degeneracy equal to the magnetic flux quanta $|k|$ defined in Eq. (5).

The lowest Landau mode in the continuum takes a Gaussian form, $\psi_{\vec{B}}(x, y) \sim e^{-|q e B|\left(x^{2}+y^{2}\right) / 4}$. It has been noted elsewhere $[9,16]$ that in a finite volume the periodicity of the lattice causes the wave function's form to be altered. We can calculate the eigenmodes of the two-dimensional (2D) Laplacian in Eq. (11) and project at the quark level. Define a projection operator onto the lowest $n$ eigenmodes $\left|\psi_{i, \vec{B}}\right\rangle$ of the 2D Laplacian as

$$
P_{n}=\sum_{i=1}^{n}\left|\psi_{i, \vec{B}}\right\rangle\left\langle\psi_{i, \vec{B}}\right| .
$$

A coordinate-space representation of this two-dimensional projection operator is applied at the sink to the quark propagator

$$
S_{n}(\vec{x}, t ; \overrightarrow{0}, 0)=\sum_{\vec{x}^{\prime}} P_{n}\left(\vec{x}, \vec{x}^{\prime}\right) S\left(\vec{x}^{\prime}, t ; \overrightarrow{0}, 0\right),
$$

where $n=\left|3 q_{f} k_{d}\right|$ modes for the lowest Landau level.

The $U(1)$ Laplacian is not QCD gauge covariant, and hence we fix the gluon field to Landau gauge and apply the appropriate gauge rotation to the quark propagator before projecting. However, as the hadronic correlation function (and ground state energy) is gauge invariant, using a gaugefixed sink operator can only effect the overlap with the ground state, which has the potential to improve the final precision of our result.

\section{One-dimensional spatial modulation}

The eigenmodes of the two-dimensional $U(1)$ Laplacian have no dependence on the $z$ coordinate. Using this freedom, we can apply a functional form to vary the spatial extent of the $U(1)$ Landau projection in the $\hat{z}$ direction, an idea analogous to standard Gaussian smearing. We modulate the $z$ dependence of the projected quark propagator with a normalized Gaussian,

$$
\phi_{\sigma}(z)=\frac{1}{\sigma \sqrt{2 \pi}} \exp \left(-\frac{z^{2}}{2 \sigma^{2}}\right),
$$

where the width parameter $\sigma \equiv \sigma_{z}$ controls the spatial extent in the $z$ direction. After the $U(1)$ Landau mode projection has been applied at the sink to the quark propagator as in Eq. (13), the gauge-fixed propagator is 
then averaged over the $z$ dimension using the modulation function as a weighting,

$S_{n, \sigma}(x, y, z, t ; \overrightarrow{0}, 0)=\sum_{z^{\prime}} \phi_{\sigma}\left(z-z^{\prime}\right) S_{n}\left(x, y, z^{\prime}, t ; \overrightarrow{0}, 0\right)$.

We define the special case $\sigma_{z}=0$ to indicate that no $z$ modulation is applied, which is equivalent to choosing $\phi_{\sigma=0}(z)=\delta\left(z^{\prime}-z\right)$, such that $S_{n, 0} \equiv S_{n}$.

Different spatial extents change the coupling to each of the energy eigenstates. The lowest lying level is dominant in the long Euclidean time limit. To determine which spatial extent provides the greatest overlap with the lowest lying energy level, many choices of $\sigma_{z}$ are investigated simultaneously [17].

The magnetic-field orientation and neutron spin polarization can be chosen independently to be in the positive or negative $z$ direction. In order to efficiently extract the magnetic polarizability, combinations of correlation functions with differing magnetic-field orientation and spin-polarization alignments are used to create spin and magnetic field aligned and antialigned correlation functions. These are the energies which will be examined in order to optimize the quark sink.

The quark sink selected is the one which has the longest plateau when fitting backward in Euclidean time from where all of the correlators agree. In evaluating this extent, the $\chi_{\text {dof }}^{2}$ is determined via a consideration of the full matrix of covariances between different time slices under consideration, and we employ an upper limit of 1.2. The sinkprojected correlator that has converged the earliest is considered optimal. This process is undertaken for each combination of field strength and aligned or antialigned energies. Figure 2 shows an example of this process for the $m_{\pi}=411 \mathrm{MeV}$ neutron and the largest magnetic field considered with $|k|=3$. It is quite clear that all the sink

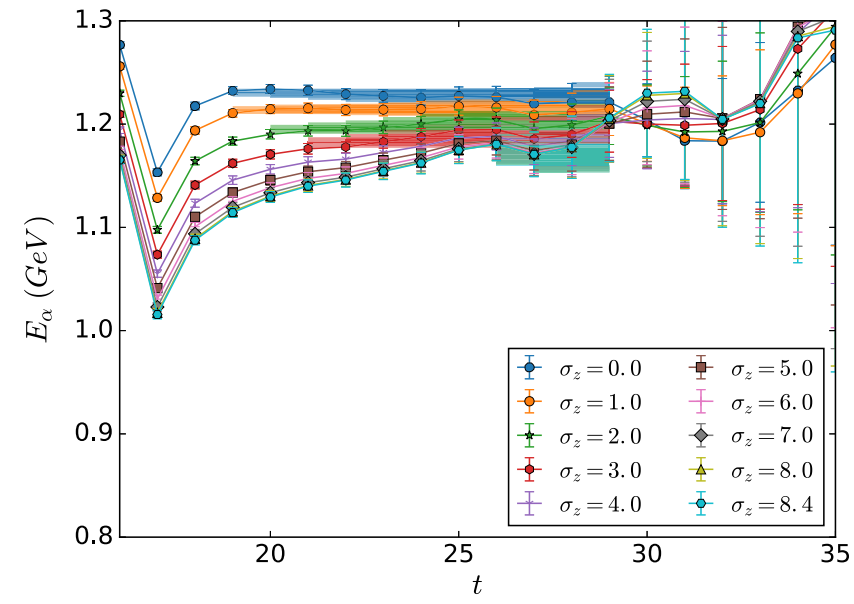

FIG. 2. Antialigned effective energy of the neutron in the largest field strength, $|k|=3$, for $U(1)$ Landau-projected sinks at $m_{\pi}=411 \mathrm{MeV}$. Consecutive fits ending at $t=29$ where all effective masses agree with $\chi_{\text {dof }}^{2} \leq 1.2$ are shown.

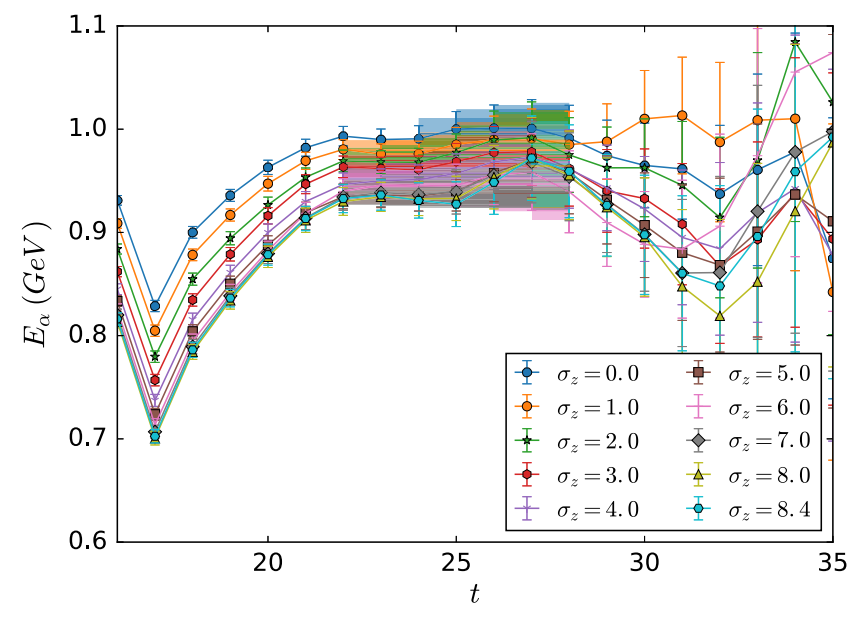

FIG. 3. Aligned effective energy of the neutron in the smallest field strength, $|k|=1$, for $U(1)$ Landau-projected sinks at $m_{\pi}=296 \mathrm{MeV}$. Consecutive fits ending at $t=28$ where all effective masses agree with $\chi_{\text {dof }}^{2} \leq 1.2$ are shown.

projections agree by $t=29$ and that $\sigma_{z}=0,1$ both produce excellent early plateaus. Figure 3 shows the aligned energies for $m_{\pi}=296 \mathrm{MeV}$ in the smallest field strength. In this case, there is no clear longest plateau. In cases like this where multiple $\sigma_{z}$ sink projections are allowed by both length and the $\chi_{\text {dof }}^{2}$, the full process for calculating the magnetic polarizability is performed for each value of $\sigma_{z}$. The resulting magnetic polarizability values are averaged to give a combined statistical error as well as a systematic error associated with the range of allowed $\sigma_{z}$.

In general, small $\sigma_{z}$ values, $\sigma_{z}=0,1,2$, are preferred across multiple pion masses, field strengths, and aligned or antialigned combinations. These sink projections provide a good representation of the neutron ground state in a background magnetic field, as can be seen by the plateau behavior in the energy of the neutron in Fig. 4.

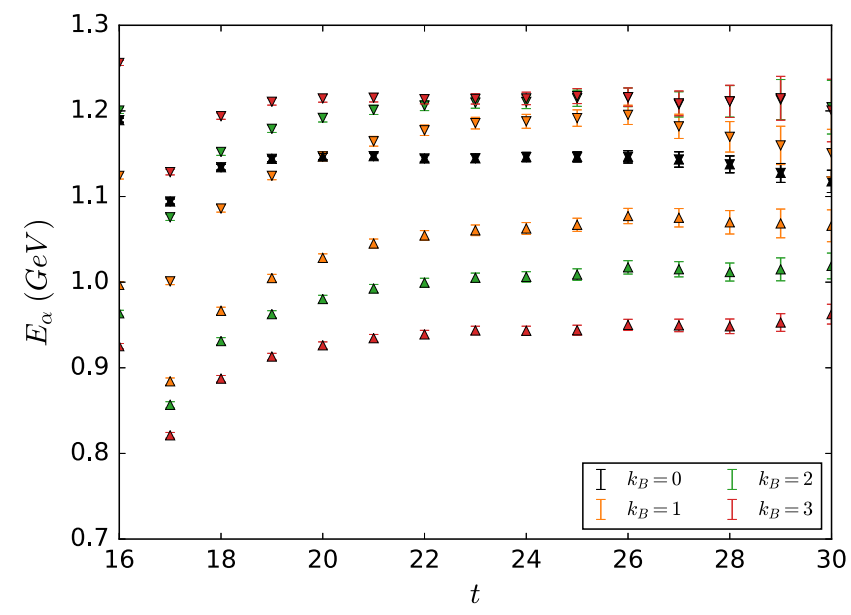

FIG. 4. Aligned (up arrows) and antialigned (down arrows) effective energies of the $m_{\pi}=411 \mathrm{MeV}$ neutron using a $U(1)$, $\sigma_{z}=1.0$ Landau mode sink projection. Three nonzero-fieldstrength energies and the zero-field mass are illustrated. 
This result represents a significant advance in the determination of magnetic polarizabilities. For the first time, clear plateaus are identified, a direct result of our consideration of Landau modes at the quark level.

\section{MAGNETIC POLARIZABILITY}

\section{A. Formalism}

Recalling the energy-field relation of Eq. (1), we note that a combination of energies at different spin orientations and field strengths can be used to isolate the neutron magnetic polarizability $\beta$,

$$
\begin{aligned}
\delta E(B) & =\frac{1}{2}\left[\left(E_{\uparrow}(B)-E_{\uparrow}(0)\right)+\left(E_{\downarrow}(B)-E_{\downarrow}(0)\right)\right] \\
& =-\frac{4 \pi}{2} \beta B^{2}+\mathcal{O}\left(B^{4}\right),
\end{aligned}
$$

noting that as $q=0$ for the neutron the Landau energy term vanishes. Here, the arrows denote the neutron spin polarization along the $\hat{z}$ axis.

This method of isolating the polarizability term is valid, but in practice due to the cancellation of correlated fluctuations on a common ensemble of lattice configurations, it is much more effective to take ratios of appropriate spin-up $(+s)$ and spin-down $(-s)$ correlators. We can also average over both positive $(+B)$ and negative $(-B)$ magnetic-field orientations to provide an improved unbiased estimator. Thus, we define the spin-field aligned correlator by

$$
G_{\Uparrow}(B)=G(+s,+B)+G(-s,-B)
$$

and the spin-field antialigned correlator by

$$
G_{1 l}(B)=G(+s,-B)+G(-s,+B) .
$$

The spin-field aligned and antialigned correlators, combined with the spin-averaged zero-field correlator, are used to form the ratio

$$
R(B, t)=\frac{G_{1 \Uparrow}(B, t) G_{1 \downarrow}(B, t)}{G(0, t)^{2}} .
$$

The product of the spin-field aligned and antialigned correlators yields an exponent that is the sum of the respective energies $\sim E_{1}+E_{1}$, removing the contribution from the magnetic moment term. Our calculation is systematically improved by including the contributions from all four field and spin pairings, such that upon taking the effective energy we obtain the desired energy shift,

$$
\begin{aligned}
\delta E(B, t) & =\frac{1}{2} \frac{1}{\delta t} \log \left(\frac{R(B, t)}{R(B, t+\delta t)}\right) \\
& =-\frac{4 \pi}{2} \beta B^{2}+\mathcal{O}\left(B^{4}\right) .
\end{aligned}
$$

Note that we define the magnetic field $\pm B$ to be that experienced by the nucleon and is hence related to the down quark magnetic field by a factor of -3 .
Any correlated QCD fluctuations between the finite field strength and zero-field effective energies are significantly reduced by taking the ratio in Eq. (19). As the zero-field correlator does not have a Landau level, the $U(1)$ eigenmode projection technique is not applied, and we use a standard point sink instead. This motivates the source tuning process outlined in Sec. III A. By using a source optimized for the zero-field neutron in the denominator of Eq. (19), the onset of plateau behavior in the effective energies occurs at an early Euclidean time. This improved method is particularly important as the polarizability is at second order in $B$, and as such at these small field strengths, its contribution to Eq. (1) is small. It is essential to have a precise determination of the polarizability energy shift. The efficiency of the Landau mode sink projection can be seen in Fig. 5 where the energy shift for a standard, point sink is compared to a $U(1)$ Landau mode sink projection; the latter is seen to display better plateau behavior.

\section{B. Simulation details}

In this work, $2+1$ flavor dynamical gauge configurations provided by the PACS-CS [18] group through the ILDG [19] are used. These have a clover fermion action and Iwasaki gauge action with a physical lattice spacing of $a=0.0907(13)$. Four values of the light quark hopping parameter $\kappa_{u d}=0.13700,0.13727,0.13754,0.13770$ are considered, corresponding to pion masses of $m_{\pi}=702$, 570, 411, $296 \mathrm{MeV}$ respectively. The lattice spacing for each mass was set using the Sommer scale with $r_{0}=0.49 \mathrm{fm}$. The lattice volume is $L^{3} \times T=32^{3} \times 64$, and the ensemble sizes are 399, 400, 449, 400 configurations respectively. Source locations were systematically varied in order to produce large distances between adjacent source locations. Starting from an initial source location at

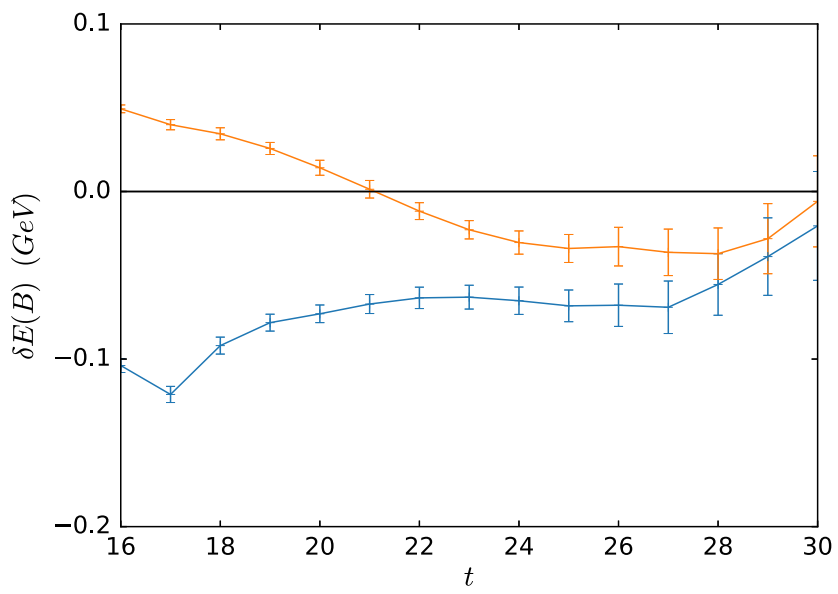

FIG. 5. The magnetic polarizability effective energy shift at the largest field strength for the neutron as a function of Euclidean time (in lattice units), using a smeared source. A point sink (orange) and a $U(1)$ Landau mode quark sink (blue) are illustrated. 
$(\overrightarrow{0}, 16)$, shifts of $(\overrightarrow{0}, 16)$ were applied three times for a total of four source locations. A further set of four shifts starting at $(\overrightarrow{16}, 8)$, where only the time component increased by 16 , were also applied. As such, a total of eight sources were used for each configuration.

Correlation functions at four distinct magnetic-field strengths are calculated. To do this, propagators at ten nonzero-field strengths, $e B= \pm 0.087, \pm 0.174, \pm 0.261$, $\pm 0.348, \pm 0.522 \mathrm{GeV}^{2}$, are calculated. These correspond to $k_{d}= \pm 1, \pm 2, \pm 3, \pm 4, \pm 6$ in Eq. (5). The zero-momentum projected correlation functions contain spin-up and spindown components.

We note that at the higher field strengths considered here one might be concerned about the validity of the energyfield expansion of Eq. (1). We can relate the energy-field expansion in Eq. (1) to the relativistic energy of a baryon in an external background magnetic field by considering $E^{2}(B)-m^{2}=(E(B)-m)(E(B)+m)$ and applying the nonrelativistic approximation $(E+m) \simeq 2 m$. Thus, $2 m /(E+m) \simeq 1$ is a measure of the importance of relativistic effects. We find $2 m /(E+m)$ to be typically within a few percent of one for all but the largest field strength. At the lightest quark mass, the effect can approach $10 \%$. However, this is a small effect in the context of the current statistical uncertainties and other systematic uncertainties discussed in the following. Still, it is an important issue to consider as one moves toward the precision era of magnetic polarizability calculations in lattice QCD. It is also important to note that these configurations are electroquenched; the field exists only for the valence quarks of the hadron. To include the background field at configuration, generation time is possible [20], but requires a separate Monte Carlo simulation for each field strength and is hence prohibitively expensive. Separate calculations also destroy the advantageous correlations between the field strengths used when constructing the ratio in Eq. (19). An alternative is to use a reweighting procedure on the gauge-field configurations [21] for the different field strengths $B$, but this is not performed here.

\section{FITTING}

The energy shift at each field strength has the form specified by Eq. (16), and as such, we fit with a quadratic term,

$$
\delta E(B, t)=\frac{4 \pi}{2} \beta B^{2}+\mathcal{O}\left(B^{4}\right) .
$$

Figures 6 and 7 show fits for the neutron energy shift with a smeared source and a $U(1)$ Landau mode sink projection. For the first time, clear plateaus are present in this difficultto-obtain quantity. It is required that a plateau be present at each of the three nonzero-field strengths in order to proceed to the next stage.

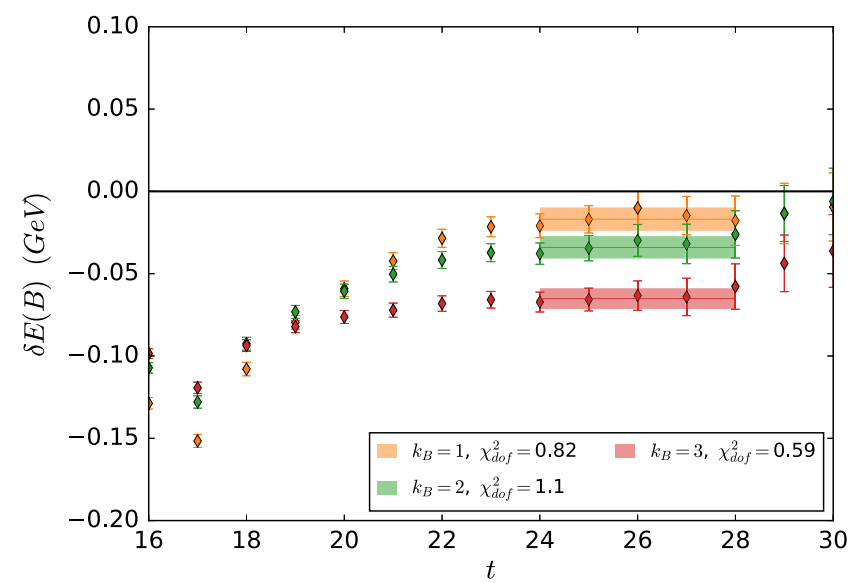

FIG. 6. The magnetic-polarizability effective-energy shift for the $m_{\pi}=411 \mathrm{MeV}$ neutron as a function of Euclidean time (in lattice units), using a smeared source and $\sigma_{z}=1.0 \mathrm{U}(1)$ Landau mode sink projection. Results for field strengths $k_{B}=1,2,3$ are shown, with the magnetic-field strength increasing away from zero. The selected fits and $\chi_{\text {dof }}^{2}$ are also illustrated.

The plateau does not occur until $t=24$; this region is a common starting point across the heavier masses. The primary cause for this late plateau onset time is the zerofield correlator, which has fundamentally different physics. As such, its potential excited state behavior is different than that of the background-field correlators. Plateaus only form once both correlators have decayed to the ground state.

The fit performed is as a function of $k_{d}$, the integer magnetic flux quanta in Eq. (5),

$$
\delta E\left(k_{d}\right)=c_{2} k_{d}^{2}
$$

Here, $c_{2}$ is the fit parameter and has units of $\mathrm{GeV}$ as these are the units of $\delta E\left(k_{d}\right)$. As a check of the validity of the

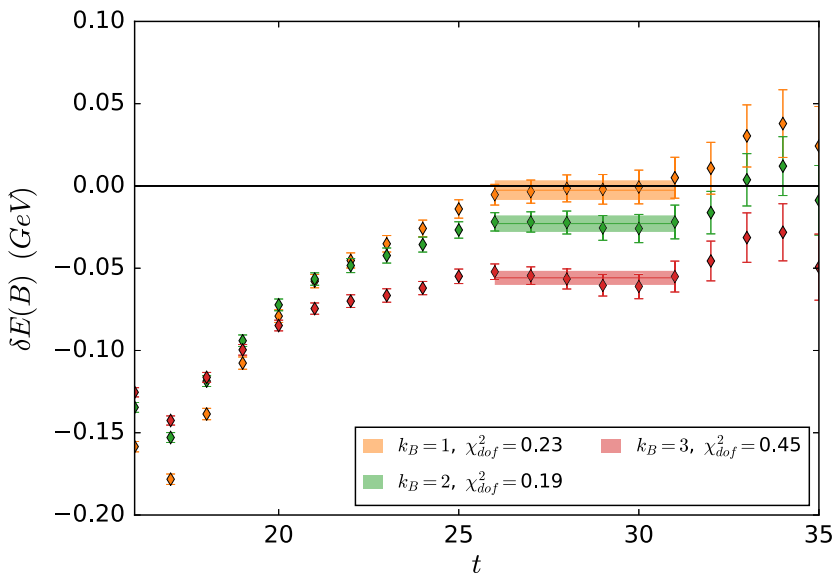

FIG. 7. The magnetic-polarizability effective-energy shift for the $m_{\pi}=702 \mathrm{MeV}$ neutron as a function of Euclidean time (in lattice units), using a smeared source and $\sigma_{z}=0.0 U(1)$ Landau mode sink projections. Symbols are as described in Fig. 6. 

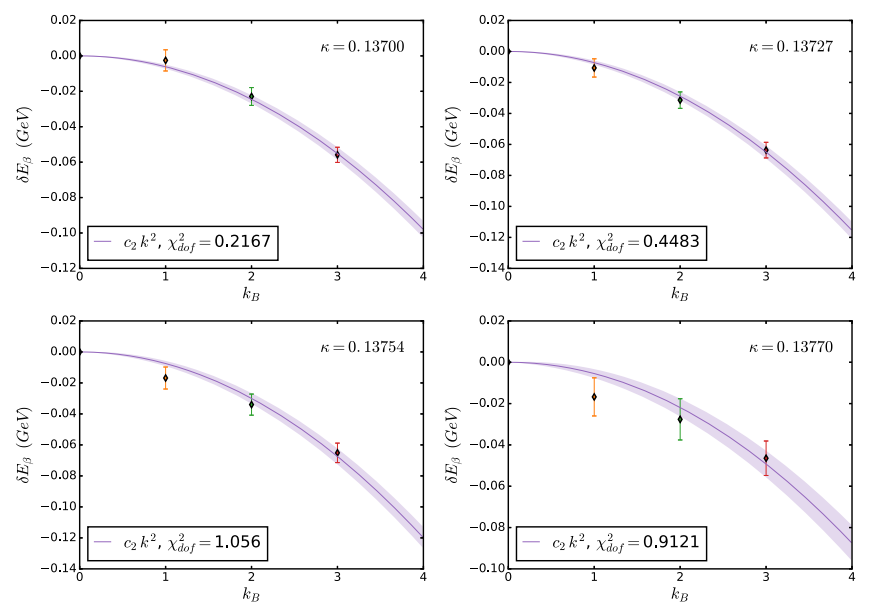

FIG. 8. Quadratic fits of the energy shift to the field quanta at each quark mass for the neutron for a single $\sigma_{z}$ value each.

expansion in Eq. (1) and hence the energy shift in Eq. (21), we also perform a quadratic + quartic fit, $c_{2} k_{d}^{2}+c_{4} k_{d}^{4}$, where the size of the quartic term provides an estimate of the corrections. It is found that for the two heavier masses, $m_{\pi}=702,570 \mathrm{MeV}$, the quartic term is indistinguishable from zero, while for $m_{\pi}=411 \mathrm{MeV}$, the fit is disfavored by the $\chi_{\text {dof }}^{2}$ of the fit. While the quadratic + quartic fit works for $m_{\pi}=293 \mathrm{MeV}$, the uncertainties are extremely large, suggesting that such a fit is only possible due to the larger uncertainties associated with lighter quark mass. If Eq. (1) were not valid at the field strengths considered herein, a remnant magnetic moment term proportional to $B$ would exist in Eq. (21). It was found that it is possible to fit a purely quadratic term as in Eq. (22) at each pion mass, and the inclusion of a quartic term is not required, confirming the validity of Eq. (1) for the neutron at the field strengths considered in this study. The quadratic fits are displayed in Fig. 8.

In order to convert this fit parameter to the physical units of magnetic polarizability, $\mathrm{fm}^{3}$, Eq. (5) is used to produce the transformation

$$
\beta=-2 c_{2} \alpha q_{d}^{2} a^{4}\left(\frac{N_{x} N_{y}}{2 \pi}\right)^{2}
$$

Here, $\alpha$ is the fine structure constant, $\alpha \approx 1 / 137$.

The quadratic fitting process uses only energy shifts which have the same spatial modulation of Eq. (14). As was seen in Figs. 2 and 3, the spatial extent affects the coupling to the energy eigenstates. It is hence important that when we fit we use the optimal sink projection. This is achieved using a simultaneous investigation of the spatial extent, $\sigma_{z}$, and field strength. For a specified spatial modulation to be suitable, it must provide early isolation of the eigenstate at each field strength. This isolation is visible in the long plateaus of Figs. 2 and 3.
TABLE I. Magnetic polarizability values for the neutron at each quark mass. Eight sources are used for each quark mass. The numbers in parentheses describe statistical and systematic uncertainties respectively.

\begin{tabular}{lccc}
\hline \hline$\kappa$ & $m_{\pi}(\mathrm{MeV})$ & $\beta\left(\mathrm{fm}^{3} \times 10^{-4}\right)$ & $\chi_{\text {dof }}^{2}$ \\
\hline 0.13700 & 702 & $1.51(21)(6)$ & 0.21 \\
0.13727 & 570 & $1.63(16)$ & 0.44 \\
0.13754 & 411 & $1.29(20)(11)$ & 1.06 \\
0.13770 & 296 & $1.14(25)(17)$ & 0.91 \\
\hline \hline
\end{tabular}

This is already a strong constraint on the sink choice, but in order to determine where to fit energy shifts for the quadratic fit in $B$, a further constraint is needed. This constraint comes from considering the constant plateau fits to the energy shift at all field strengths. By considering all possible fit windows, we select fit windows where good plateau behavior exists for all field strengths simultaneously. Good plateau behavior is characterized by a fit $\chi_{\text {dof }}^{2}$ of less than 1.2. This process of requiring good plateau behavior at each field strength simultaneously dramatically reduces the number of possible fit windows. In particular, it is often difficult to obtain acceptable energy shift plateaus for the largest field strength considered.

The final constraint on the fitting process comes from the quadratic fit itself. This fit must also be acceptable having a $\chi_{\text {dof }}^{2} \leq 1.2$. If multiple fit windows remain after this process, the one with the longer time extent and $\chi_{\text {dof }}^{2}$ 's closest to one are preferred.

Once the specific quadratic fit has been chosen, the magnetic polarizability, $\beta$, is extracted from the quadratic coefficient of the fit. In order to test the presence of higherorder terms in the energy shift of Eq. (21), a quartic term is also considered. It is found that the quartic term is not needed in order to fit the energy shifts well with acceptable $\chi_{\text {dof }}^{2}$.

Using the sink eigenmode-projection technique at each quark mass, it is possible to extract magnetic polarizabilities from the fits to the constant energy shift plateaus as a function field strength. Results are presented in Table I for the magnetic polarizability of the neutron at each quark mass. Note that for the $m_{\pi}=570 \mathrm{MeV}$ ensemble no systematic error due to the choice of $\sigma_{z}$ is reported as only $\sigma_{z}=0$ provided good access to the ground state across all field strengths.

\section{CHIRAL EXTRAPOLATION}

\section{A. Formalism}

Chiral effective-field theory ( $\chi \mathrm{EFT})$ is an important tool for connecting lattice results to the physical point. The analysis here follows that of Ref. [22] and is summarized briefly below. 
We consider the chiral expansion

$$
\beta\left(m_{\pi}^{2}\right)=\beta^{\pi N}\left(m_{\pi}^{2}\right)+\beta^{\pi \Delta}\left(m_{\pi}^{2}\right)+a_{0}+a_{2} m_{\pi}^{2} .
$$

The leading-order loop contributions $\beta^{\pi N}\left(m_{\pi}^{2}\right)$ and $\beta^{\pi \Delta}\left(m_{\pi}^{2}\right)$ are depicted in Figs. 9 and 10. The loop integral of Eq. (25) for $\beta^{\pi N}\left(m_{\pi}^{2}\right)$ contains the leading nonanalytic contribution to the chiral expansion proportional to $1 / m_{\pi}$ [23]. Similarly, the integral of Eq. (26) for $\beta^{\pi \Delta}\left(m_{\pi}^{2}\right)$ accounts for transitions to a Delta baryon. For a finite nucleon-Delta mass splitting, $\Delta=M_{\Delta}-M_{N}$, this diagram contributes a nonanalytic logarithmic contribution proportional to $(-1 / \Delta) \log \left(m_{\pi} / \Lambda\right)$ to the expansion. Here, $\Lambda$ is a renormalization scale. The coefficients $a_{0}$ and $a_{2}$ are residualseries coefficients [24] which will be constrained by our lattice QCD results after they are corrected to infinite volume. Once combined with the analytic contributions contained in the loop integrals [25], these parameters form the renormalized low-energy coefficients of the chiral expansion. Complete details of the renormalization procedure are provided in the Appendix of Ref. [25].

The loop-integral contributions $\beta^{\pi N}\left(m_{\pi}^{2}\right)$ and $\beta^{\pi \Delta}\left(m_{\pi}^{2}\right)$ are evaluated in the heavy-baryon limit [26] appropriate to a low-energy expansion. The three-dimensional integral forms are [22]

$$
\begin{aligned}
\beta^{\pi N}\left(m_{\pi}^{2}\right) & =\frac{e^{2}}{4 \pi} \frac{1}{288 \pi^{3} f_{\pi}^{2}} \chi_{N} \int \mathrm{d}^{3} k \frac{\vec{k}^{2} u^{2}(k, \Lambda)}{\left(\vec{k}^{2}+m_{\pi}^{2}\right)^{3}}, \\
\beta^{\pi \Delta}\left(m_{\pi}^{2}\right)= & \frac{e^{2}}{4 \pi} \frac{1}{288 \pi^{3} f_{\pi}^{2}} \chi_{\Delta} \int \mathrm{d}^{3} k u^{2}(k, \Lambda) \\
& \times \frac{\omega_{\vec{k}}^{2} \Delta\left(3 \omega_{\vec{k}}+\Delta\right)+\vec{k}^{2}\left(8 \omega_{\vec{k}}^{2}+9 \omega_{\vec{k}} \Delta+3 \Delta^{2}\right)}{8 \omega_{\vec{k}}^{5}\left(\omega_{\vec{k}}+\Delta\right)^{3}} .
\end{aligned}
$$

Here, $\omega_{\vec{k}}=\sqrt{\vec{k}^{2}+m_{\pi}^{2}}$ is the energy carried by the pion which has three-momentum $\vec{k}, \Delta$ is the aforementioned mass splitting between the Delta baryon and the nucleon, $\Delta \equiv M_{\Delta}-M_{N}=292 \mathrm{MeV}$, and the pion decay constant is taken as $f_{\pi}=92.4 \mathrm{MeV}$. The dipole regulator

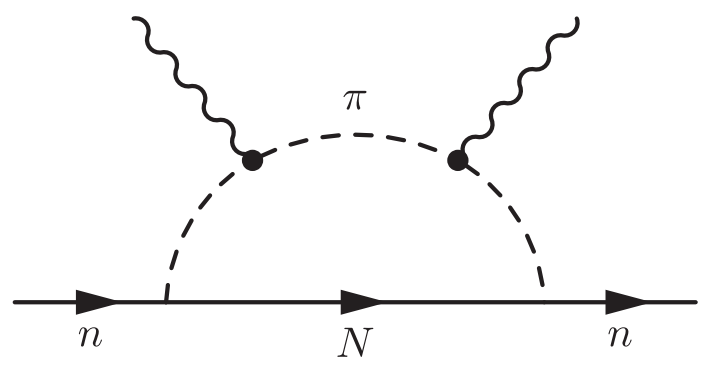

FIG. 9. The leading-order pion-loop contribution to the magnetic polarizability of the neutron.

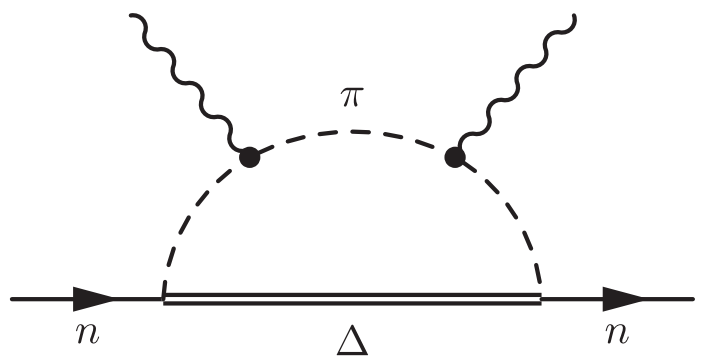

FIG. 10. Pion-loop contributions to the magnetic polarizability of the neutron, allowing transitions to the nearby and strongly coupled $\Delta$ baryons.

$$
u(k, \Lambda)=\frac{1}{\left(1+\vec{k}^{2} / \Lambda^{2}\right)^{2}}
$$

of Eqs. (25) and (26) ensures that only soft momenta flow through the effective-field theory degrees of freedom.

The lattice QCD results do not incorporate contributions from photons coupling to the disconnected sea-quark loops of the vacuum which form the full meson dressings of $\chi$ EFT - they are electroquenched. Thus, it is necessary to model the corrections associated with these effects. This is done using partially quenched $\chi$ EFT. In this case, the standard coefficients for full QCD,

$$
\begin{gathered}
\chi_{N}=2 g_{A}^{2}, \\
\chi_{\Delta}=\frac{16}{9} \mathcal{C}^{2},
\end{gathered}
$$

are modified to account for partial quenching effects [27] as explained in Ref. [22]. Thus, when fitting the lattice QCD results, we use coefficients that reflect the absence of disconnected sea-quark-loop contributions.

$$
\begin{gathered}
\chi_{N} \rightarrow \chi_{N}^{p Q}=2 g_{A}^{2}-(D-F)^{2}-\frac{7}{27}(D+3 F)^{2}, \\
\chi_{\Delta} \rightarrow \chi_{\Delta}^{p Q}=\frac{16}{9} \mathcal{C}^{2}-\frac{2}{9} \mathcal{C}^{2} .
\end{gathered}
$$

We use the standard values of $g_{A}=1.267$ and $\mathcal{C}=-1.52$ with $g_{A}=D+F$ and the $S U(6)$ symmetry relation $F=\frac{2}{3} D$.

In anticipation of accounting for the missing disconnected sea-quark-loop contributions in the lattice QCD calculations, the value $\Lambda=0.80 \mathrm{GeV}$ is adopted [28-32]. This regulator mass defines a pion cloud contribution to masses [29], magnetic moments [30], and charge radii [28], which enables corrections to the pion cloud contributions associated with missing disconnected sea-quark-loop contributions. This particular choice of regulator mass defines a neutron core contribution insensitive to sea-quark-loop contributions [33]. 
Finite-volume effects are considered by replacing the continuum integrals of the chiral expansion with sums over the momenta available on the periodic lattice. We note that the lattice volume varies slightly across the four lattice data points available due to our use of the Sommer scale.

\section{B. Analysis}

We proceed by calculating the integrals of Eqs. (25) and (26) in the finite volume of the periodic lattice by replacing the continuum integrals of the chiral expansion with sums over the momenta available. As the lattice QCD results do not include the contributions of disconnected sea-quarkloop contributions, the coefficients of Eqs. (30) and (31) are used in Eqs. (25) and (26). This calculation is carried out at each quark mass considered on the lattice.

One then numerically integrates Eqs. (25) and (26) in infinite volume and with the full QCD coefficients of Eqs. (28) and (29). The difference between this infinitevolume full-QCD result and aforementioned finite-volume partially quenched result at each quark mass is used to correct the lattice QCD results to infinite volume and full QCD. In this way, both finite-volume and sea-quarkloop contribution corrections are incorporated. These corrections are illustrated in Fig. 11 by the (blue square) "Full-QCD Infinite-Volume Results" next to the original (violet-diamond) "Lattice Results."

At this point, the fit function of Eq. (24) is fit to the corrected lattice QCD results by adjusting the residualseries coefficients, $a_{0}$ and $a_{2}$. Once $a_{0}$ and $a_{2}$ are constrained, any volume can be considered. Figure 11 shows chiral extrapolations for a range of volumes to provide guidance to future lattice QCD simulations. Large box sizes

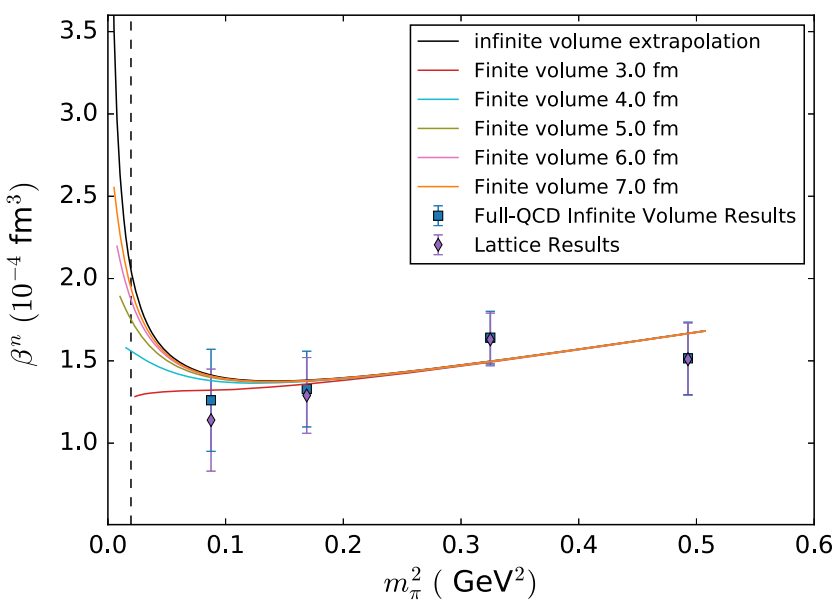

FIG. 11. Correction of the lattice QCD results (violet diamond) for the neutron magnetic polarizability $\beta^{n}$ to infinite volume and full QCD (blue square) as described in the text. Extrapolations of $\beta^{n}$ for a variety of spatial lattice volumes provide a guide to future lattice QCD simulations. The infinite-volume case relevant to experiment is also illustrated. are required in order to obtain an extrapolation close to the infinite-volume value at the physical point.

The physical polarizability is obtained from the constrained fit function of Eq. (24) with $m_{\pi}=m_{\pi}^{\text {phys }}=$ $140 \mathrm{MeV}$. While the coefficients of the leading nonanalytic terms of the chiral expansion are determined in a modelindependent manner, uncertainty in the higher-order terms of the expansion can be examined through a variation of the regulator parameter $\Lambda$, which affects the sum of these contributions. Consideration of the broad range of $0.6 \leq \Lambda \leq 1.0 \mathrm{GeV}$ provides a systematic uncertainty of $0.19 \times 10^{-3} \mathrm{fm}^{3}$ at the physical point. Thus, we find $\beta^{n}=2.05(25)(19) \times 10^{-4} \mathrm{fm}^{3}$ at the physical point. The uncertainties are derived from the statistical errors of the fit parameters and the systematic uncertainty associated with the chiral extrapolation respectively.

A comparison between this result and the experimental data is provided in Fig. 12. Our calculation is in good agreement with a number of the experimental results and poses an interesting challenge for greater experimental precision. Similarly, progress in experimental measurement would drive further lattice QCD and chiral effective-field theory work.

These lattice results use a single lattice spacing, and as such, it is not possible to quantify an uncertainty associated with taking the continuum limit. However, as a nonperturbatively improved clover fermion action is used, the $\mathcal{O}\left(a^{2}\right)$ corrections are expected to be small relative to the uncertainties already presented. It is anticipated that there is some degree of additive quark mass renormalization due to the interaction of the background field with the Wilson term in the fermion action [35], and the extent to

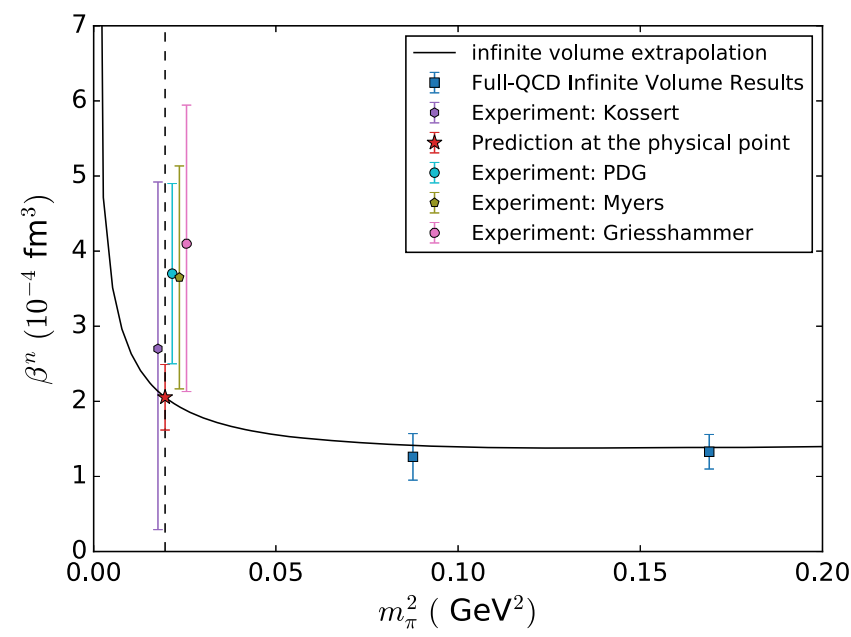

FIG. 12. The magnetic polarizability of the neutron, $\beta_{n}$ obtained herein is compared with experimental results. The uncertainties in the lattice results contain both statistical and systematic errors simply added together. This is a conservative approach to produce a reliable estimation. Experimental results from Griesshammer et al. [3], the PDG [34], Kossert et al. [1,2], and Myers et al. [4] are offset for clarity. 
which this small effect remains with the clover fermion action is under investigation [36].

\section{CONCLUSION}

The neutron magnetic polarizability has been calculated using a novel approach in which asymmetric operators are used at the source and sink. The use of gaugeinvariant Gaussian smearing at the source encapsulates the dominant QCD dynamics, while a gauge-fixed $U(1)$ two-dimensional eigenmode projection technique is used at the sink to encode the Landau level physics resulting from the presence of the uniform magnetic field. A systematic exploration of the parameter space was used to optimize operators that couple efficiently to the neutron ground state in a magnetic field. The use of this Landau mode projection at the sink has for the first time enabled the fitting of plateaus in the magnetic polarizability energy shifts.

Calculations at several pion masses have enabled the use of heavy-baryon chiral effective-field theory to relate the lattice QCD results to experiment. This enables us to make a theoretical prediction for the neutron magnetic polarizability of $\beta^{n}=2.05(25)(19) \times 10^{-4} \mathrm{fm}^{3}$. This prediction is founded on $a b$ initio lattice QCD simulations, with effective-field theory techniques used to account for the finite-volume of the lattice, disconnected sea-quark-loop contributions and an extrapolation to the light quark masses of nature. The resulting value is in agreement with the current experimental estimates and presents an interesting challenge for greater experimental precision.

A range of finite-volume extrapolations is performed in the framework of chiral effective-field theory. As these curves incorporate the contributions of sea-quark loops, they are presented as a guide to future lattice QCD simulations. At the physical pion mass, the $7 \mathrm{fm}$ curve still differs from the infinite-volume prediction by $6 \%$.

Our result does not directly incorporate the sea-quarkloop effects of the magnetic field in the lattice simulation, which would require a separate Monte Carlo ensemble for each value of $B$ considered and as such is prohibitively expensive. We also note that in this study we have not considered the effects from the $B$-dependent additive quark mass renormalization that arises due to the use of Wilsontype fermions [35]. The detailed impact of this effect on clover fermions has not been studied and will be addressed in a separate work that is in preparation [36]. While the effect can be observed in pion correlators, our preliminary tests indicate it is hidden by the large statistical fluctuations inherent in baryon correlators.
It is interesting to consider that, in obtaining the magnetic polarizability, we want to work with small $\vec{B}$ field strengths in order to make use of the perturbative energy expansion for the neutron. This means we are the confining phase of QCD, such that quarks cannot have individual Landau levels. Nonetheless, the success of our Landau mode sinks indicates that the effects of the magnetic field on the quark distribution in the neutron are significant.

Future work will examine the effect of using a gaugecovariant sink projection based on the eigenmodes of the two-dimensional $U(1) \times S U(3)$ Laplacian [15]. This alters the Landau level structure, breaking the degeneracy and mixing different levels. Indeed, a recent finite temperature study using the staggered quark formulation found that the contribution of the lowest Landau level eigenmodes remains important even after QCD interactions are introduced [14], further motivating our investigation of the effectiveness of an eigenmode-projected sink which is aware of the QCD gauge field.

Another potential avenue for future investigation would be to explore relativistic corrections to the energy-field expansion of Eq. (1). To move beyond the use of Eq. (1) requires one to separate the ratio of correlators in Eq. (19) to fit spin-field aligned and antialigned correlators separately. It will be interesting to examine the behavior of these correlators and the extent to which QCD correlations can be exploited to obtain accurate fits for the energies $E(B)+m$ and $E(B)-m$. These considerations may be particularly important in the study of charged hadrons.

\section{ACKNOWLEDGMENTS}

We thank the PACS-CS Collaboration for making their $2+1$ flavor configurations available and the ongoing support of the International Lattice Data Grid (ILDG). This work was supported with supercomputing resources provided by the Phoenix HPC service at the University of Adelaide. This research was undertaken with the assistance of resources from the National Computational Infrastructure (NCI). NCI resources were provided through the National Computational Merit Allocation Scheme, supported by the Australian Government and the University of Adelaide Partner Share. R. B. was supported by an Australian Government Research Training Program Scholarship. This research is supported by the Australian Research Council through Grants No. DP150103164 and No. DP120104627 (D. B. L.). 
[1] K. Kossert et al., Phys. Rev. Lett. 88, 162301 (2002).

[2] K. Kossert et al., Eur. Phys. J. A 16, 259 (2003).

[3] H. W. Griesshammer, J. A. McGovern, D. R. Phillips, and G. Feldman, Prog. Part. Nucl. Phys. 67, 841 (2012).

[4] L. S. Myers et al. (COMPTON@MAX-lab Collaboration), Phys. Rev. Lett. 113, 262506 (2014).

[5] J. Smit and J. C. Vink, Nucl. Phys. B286, 485 (1987).

[6] G. Martinelli, G. Parisi, R. Petronzio, and F. Rapuano, Phys. Lett. 116B, 434 (1982).

[7] M. Burkardt, D. B. Leinweber, and X. Jin, Phys. Lett. B 385, 52 (1996).

[8] C. W. Bernard, T. Draper, K. Olynyk, and M. Rushton, Phys. Rev. Lett. 49, 1076 (1982).

[9] B. C. Tiburzi and S. O. Vayl, Phys. Rev. D 87, 054507 (2013).

[10] T. Primer, W. Kamleh, D. Leinweber, and M. Burkardt, Phys. Rev. D 89, 034508 (2014).

[11] E. Chang, W. Detmold, K. Orginos, A. Parreno, M. J. Savage, B. C. Tiburzi, and S. R. Beane (NPLQCD Collaboration), Phys. Rev. D 92, 114502 (2015).

[12] C. Itzykson and J. B. Zuber, Quantum Field Theory, International Series in Pure and Applied Physics (McGraw-Hill, New York, 1980).

[13] G. Parisi, Phys. Rep. 103, 203 (1984).

[14] F. Bruckmann, G. Endrodi, M. Giordano, S. D. Katz, T. G. Kovacs, F. Pittler, and J. Wellnhofer, Phys. Rev. D 96, 074506 (2017).

[15] W. Kamleh, R. Bignell, D. B. Leinweber, and M. Burkardt, EPJ Web Conf. 175, 05018 (2018).

[16] R. Bignell, D. Leinweber, W. Kamleh, and M. Burkardt, Proc. Sci., INPC2016 (2017) 287.

[17] M. S. Mahbub, W. Kamleh, D. B. Leinweber, P. J. Moran, and A. G. Williams (CSSM Lattice Collaboration), Phys. Lett. B 707, 389 (2012).

[18] S. Aoki et al. (PACS-CS Collaboration), Phys. Rev. D 79, 034503 (2009).
[19] M. G. Beckett, B. Joo, C. M. Maynard, D. Pleiter, O. Tatebe, and T. Yoshie, Comput. Phys. Commun. 182, 1208 (2011).

[20] H. R. Fiebig, W. Wilcox, and R. M. Woloshyn, Nucl. Phys. B324, 47 (1989).

[21] W. Freeman, A. Alexandru, M. Lujan, and F. X. Lee, Phys. Rev. D 90, 054507 (2014).

[22] J. M. M. Hall, D. B. Leinweber, and R. D. Young, Phys. Rev. D 89, 054511 (2014).

[23] V. Lensky and V. Pascalutsa, Eur. Phys. J. C 65, 195 (2010).

[24] D. B. Leinweber, A. W. Thomas, and R. D. Young, Phys. Rev. Lett. 92, 242002 (2004).

[25] R. D. Young, D. B. Leinweber, and A. W. Thomas, Prog. Part. Nucl. Phys. 50, 399 (2003).

[26] E. E. Jenkins and A. V. Manohar, Phys. Lett. B 255, 558 (1991).

[27] W. Detmold, B. C. Tiburzi, and A. Walker-Loud, Phys. Rev. D 73, 114505 (2006).

[28] P. Wang, D. B. Leinweber, A. W. Thomas, and R. D. Young, Phys. Rev. D 79, 094001 (2009).

[29] R. D. Young, D. B. Leinweber, A. W. Thomas, and S. V. Wright, Phys. Rev. D 66, 094507 (2002).

[30] D. B. Leinweber, S. Boinepalli, I. C. Cloet, A. W. Thomas, A. G. Williams, R. D. Young, J. M. Zanotti, and J. B. Zhang, Phys. Rev. Lett. 94, 212001 (2005).

[31] D. B. Leinweber, S. Boinepalli, A. W. Thomas, P. Wang, A. G. Williams, R. D. Young, J. M. Zanotti, and J. B. Zhang, Phys. Rev. Lett. 97, 022001 (2006).

[32] P. Wang, D. B. Leinweber, A. W. Thomas, and R. D. Young, Phys. Rev. C 79, 065202 (2009).

[33] P. Wang, D. B. Leinweber, and A. W. Thomas, Phys. Rev. D 89, 033008 (2014).

[34] C. Patrignani et al. (Particle Data Group Collaboration), Chin. Phys. C 40, 100001 (2016).

[35] G. S. Bali, B. B. Brandt, G. Endrodi, and B. Glaessle, Phys. Rev. D 97, 034505 (2018).

[36] R. Bignell, W. Kamleh, and D. Leinweber, Pion in a uniform background field (to be published). 